\title{
Cosmic rays beyond the boundary of the heliosphere
}

\author{
V. Florinski* \\ Department of Space Science and Center for Space Plasma and Aeronomic Research, University \\ of Alabama in Huntsville, Huntsville AL 35899, USA \\ E-mail: vaf0001@uah.edu
}

\section{E. C. Stone, A. C. Cummings}

Space Radiation Laboratory, California Institute of Technology, Pasadena, CA 91125, USA

\section{J. A. le Roux}

Department of Space Science and Center for Space Plasma and Aeronomic Research, University of Alabama in Huntsville, Huntsville AL 35899, USA

\begin{abstract}
In August of 2012 the Voyager 1 space probe has left the solar-wind bubble of ionized gas we call the heliosphere and entered the denser and colder environment of the interstellar cloud surrounding the solar system. Energetic charged particles underwent dramatic changes past the heliopause: the heliospheric ions disappeared completely, while the galactic cosmic rays were for the first time measured in their unmodulated state. The interstellar medium turned out to be almost entirely devoid of turbulent magnetic fluctuations, therefore the transport of cosmic rays is governed by a large-scale geometry of the magnetic field. We discuss observations of heliospheric ions, including anomalous cosmic rays, near the heliopause transition, and propose interpretations of the measured intensities and pitch-angle distributions based on gradient drift in a weakly nonuniform magnetic field. The heliopause transition appears to be permeated by magnetic flux tubes connected to the interstellar space and facilitating particle escape. These flux tubes may be a product of interchange instability driven by a plasma pressure gradient across the heliopause. The curvature of magnetic field lines and the anti-sunward gradient in plasma kinetic pressure provide conditions favorable for an interchange. The two flux tube crossings by the spacecraft allowed an indirect measurement of the plasma radial velocity near the heliopause.
\end{abstract}

The 34th International Cosmic Ray Conference,

30 July- 6 August, 2015

The Hague, The Netherlands

\footnotetext{
* Speaker.
} 


\section{Introduction}

The heliopause is a pressure equilibrium boundary of the heliosphere, a giant "bubble" in space filled with plasma from the sun and surrounded by the cold and relatively dense interstellar cloud. At this time, it is generally agreed that the Voyager 1 space probe has crossed this boundary near 20012.65, and has been traveling through local interstellar medium (LISM) since then [1, 2, 3, 4]. The key observations in favor of the crossing were (a) a rapid depletion of heliospheric ions, evidently as a result of their escape into interstellar space, (b) an increase in galactic cosmic rays followed by virtually constant intensity for the next two years, (c) a change in the direction and strength of the magnetic field by about $20^{\circ}$ relative to the primarily azimuthal field in the heliosphere, (d) detection of plasma waves at frequencies indicating a plasma density of the order of 0.08 $\mathrm{cm}^{-3}$, some 30 times the density in the inner heliosheath (the region between the heliopause and the termination shock), and (e) a near absence of magnetic fluctuation characteristic of the interstellar medium [5]. However, the rotation of the magnetic field occurred gradually before Voyager crossed the energetic particle escape boundary, known as the heliocliff.

While still in the inner heliosheath, Voyager 1 crossed two particle dropout regions with a stronger magnetic field. The boundaries of the regions exhibited only a minimal change in the magnetic field direction [6]. In the models of [7, 8] a direct route for particle escape was created via magnetic reconnection between the heliospheric and the interstellar fields. An alternative view [1, 9] is that the regions are magnetic flux tubes sunk into the inner heliosheath through an interchange instability of the heliopause and connected to the LISM at both ends. In this paper we further develop this idea by performing a linear stability analysis of the surface of the heliopause.

The heliospheric particles developed large anisotropies in the direction normal to the mean field, i.e., only the gyrating ions remained past the heliocliff. The escape of high-rigidity oxygen ions was much more gradual than that of anomalous hydrogen and helium that have a smaller Larmor radius [2]. As proposed in [10], gradient drift in a weakly nonuniform field (lengthscale of variation $\sim 10 \mathrm{AU}$ ) efficiently differentiates particles based on their regidity, which explains why the gyrating oxygen ions did not fully disappear until about 1 AU past the heliocliff. The two particle dropouts can be explained by the spacecraft crossing the boundaries of the flux tubes. In the dropouts $5 \mathrm{MeV}$ proton intensities decreased by a factor of $\sim 4$, which requires that these particle undergo virtually no pitch-angle scattering inside the flux tubes. The rapid particle drop and recovery at the boundaries of the flux tubes can be used to estimate the speed of the spacecraft relative to the background medium and thus deduce the plasma velocity that Voyager 1 does not measure directly because of a faulty plasma instrument.

\section{Stability of the heliopause}

In the upwind-facing direction (relative to the flow of the interstellar gas past the sun) the heliopause may be locally represented as a spherical segment with a radius equal to the distance of Voyager 1 crossing (122 AU). This geometry may be tested for stability using the standard techniques developed for cylindrical plasmas in the fusion confinement community [11]. Without getting into too much detail, the MHD system of equations is linearized about the equilibrium state (with zero plasma velocity) and the perturbed system for one Fourier mode $(\omega, \mathbf{k})$ reduced to a single second- 
order differential equation for the radial component of the perturbed velocity $u_{r}$

$$
\frac{A_{u p}}{r^{2}} \frac{d}{d r}\left(\frac{r^{2}}{A_{u p}} \frac{d u_{r}}{d r}\right)+\left(A_{u u} \frac{d \ln A_{u p}}{d r}-\frac{d A_{u и}}{d r}+\operatorname{det} A\right) u_{r}=0
$$

where

$$
\begin{gathered}
A_{u u}=-\frac{2 \omega^{2} c_{s}^{2}}{r\left(\omega^{2} v_{a}^{2}+\Omega_{a}^{2} c_{s}^{2}\right)}, \quad A_{p p}=-\frac{2}{r}-A_{u u}, \\
A_{u p}=\frac{i \omega\left(\omega^{2}-k^{2} v_{f}^{2}\right)\left(\omega^{2}-k^{2} v_{s}^{2}\right)}{\rho_{0} \Omega_{a}^{2}\left(\omega^{2} v_{a}^{2}+\Omega_{a}^{2} c_{s}^{2}\right)}, \\
A_{p u}=\frac{i}{\omega}\left(\rho_{0} \Omega_{a}^{2}-\frac{2}{r} \frac{d p_{0}}{d r}\right)-\frac{i 4 \omega \rho_{0} c_{s}^{2} v_{a}^{2}}{r^{2}\left(\omega^{2} v_{a}^{2}+\Omega_{a}^{2} c_{s}^{2}\right)} .
\end{gathered}
$$

Here $\rho_{0}$ and $p_{0}$ are the equilibrium values of the plasma density and pressure, respectively, $c_{s}, v_{a}$, $v_{s}$, and $v_{f}$ are the wave speeds (sound, Alfvén, slow, and fast, respectively), and $\Omega_{a}^{2}=\omega^{2}-\left(\mathbf{k} \cdot \mathbf{v}_{a}\right)^{2}$. The wavevector $\mathbf{k}$ is tangent to the sphere.

The system (2.1)-(2.4) is integrated numerically over a radial interval much larger than the width of the heliopause transition in order to find the eigenfunctions that satisfy the zero boundary conditions far away from the heliopause. There are usually an infinite number of eigenfunctions, but the one with zero radial nodes is always the most unstable, if it is unstable at all. Across the transition the plasma pressure decreases and the magnetic field increases, so that the plasma beta drops from 5.5 in the inner heliosheath to 0.36 in the LISM, based on Voyager observations and models $[3,12]$. This configuration may become unstable and develop interchange perturbations because the curvature of magnetic field lines is directed into the region of high beta (sunward). The growth rate evidently depends on the propagation direction, the width of the transition, and the amount of magnetic shear from the misalignment of the fields on the two sides of the heliopause.

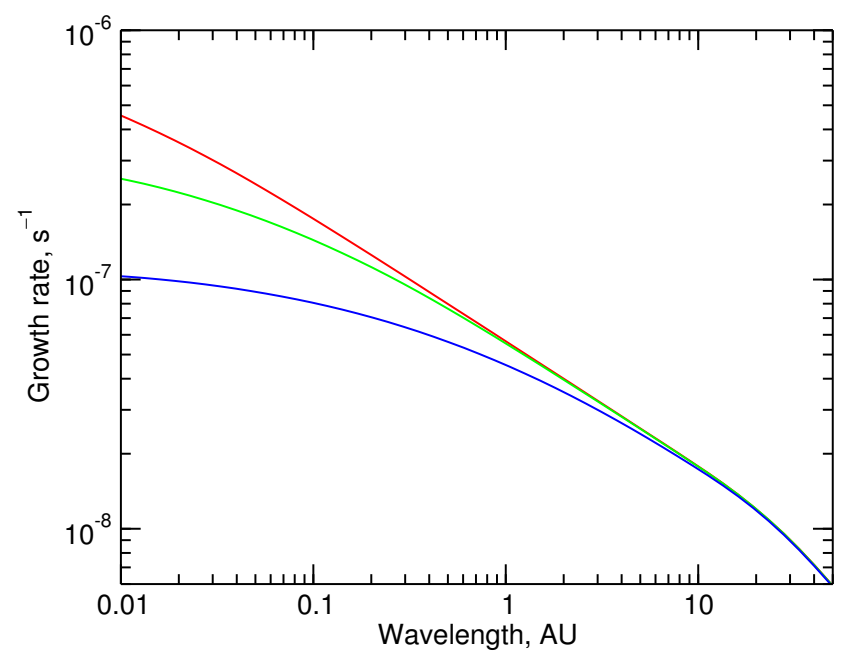

Figure 1: Theoretical linear growth rates at normal propagation for transition layer width of $0.01 \mathrm{AU}$ (red). $0.1 \mathrm{AU}$ (green), and $1 \mathrm{AU}$ (blue).

In almost all cases studied the growth rate is maximized for perpendicular propagation (pure interchange involving no bending of magnetic lines of force). We show the dependence of the instability growth rate on wavelength for the transition widths of $0.01 \mathrm{AU}, 0.1 \mathrm{AU}$, and $1 \mathrm{AU}$ in Figure 1. At the right end of the figure, the growth rates for short waves corresponds to an 
exponential growth time of about 2 months, which is fast compared to the typical dynamic timescale in the region. Because large $k_{\perp}$ is favored, the interchange will develop as thin and long $k_{\|} \rightarrow 0$ for normal propagation) filaments, consistent with the diameter of the flux tubes encountered by Voyager 1.

The shear (rotation) of magnetic field across the heliopause would tend to weaken the instability reducing the growth rate. Our analysis, which will be reported in a separate publication, shows that the heliopause remains unstable for a shear of up to $8^{\circ}$ for a $0.1 \mathrm{AU}$ wide transition. The threshold can be relaxed if the transition is wider or the radius of curvature smaller, perhaps as a result of a fold or protrusion developed on the heliopause. In that case the heliopause remains unstable for the field rotation measured by Voyager $1\left(14^{\circ}\right)$. The transition region may look like that depicted in Figure 2, where the flux tubes from the interstellar medium become immersed in the inner heliosheath plasma. The Voyager 1 path is shown crossing two of the tubes. Note that interchange is only possible if the field lines inside and outside the tubes are parallel to each other; the instability therefore may provide a mechanism for aligning the field locally with a slow rotation between the equilibrium states far away from the transition region. In the direction of Voyager 2, however, the angle of rotation is larger and the heliopause is therefore likely to be stable against magnetic interchange.

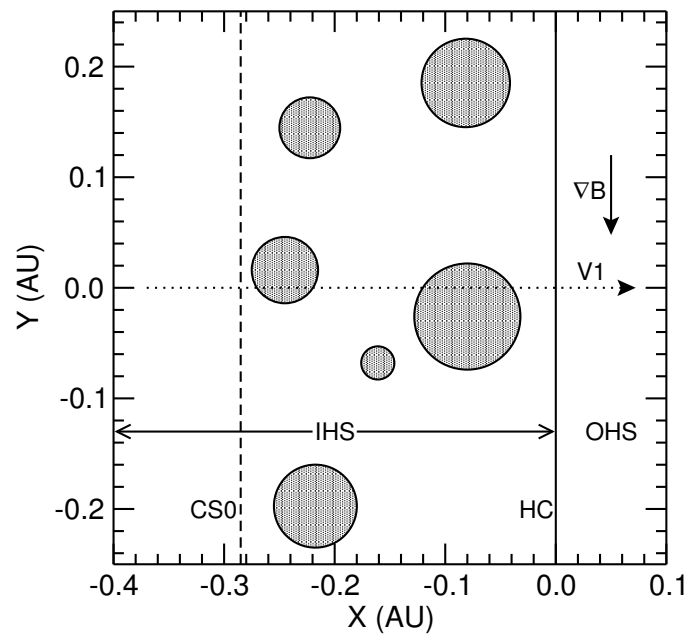

Figure 2: A conceptual drawing of the heliopause transition layer showing multiple interstellar magnetic flux tubes immersed in the inner heliosheath plasma. CSO is the last current sheet observed by Voyager 1 before leaving the heliosphere.

\section{Anomalous cosmic rays}

This section is based on our previously published results [10]. We assume the transition region geometry shown in Figure 2. Trajectories of the Boltzmann equation for the charged particles are integrated backward in time, from the "detection" point and until the trajectory leaves the computational box. Written in terms of the phase space density $f(\mathbf{r}, \mathbf{v})$, the equation we solve is

$$
\frac{\partial f}{\partial t}+\mathbf{v}_{\|} \cdot \nabla_{\|} f+\left(\mathbf{v}_{\perp}-\left|v_{\|}\right| \nabla_{\perp} K\right) \cdot \nabla_{\perp} f-\left|v_{\|}\right| K \nabla_{\perp}^{2} f-\Omega \frac{\partial f}{\partial \varphi}-D \nabla_{\theta \varphi}^{2} f=0,
$$

where $\varphi$ is gyrophase, $\nabla_{\|}$and $\nabla_{\perp}$ are the gradient operators in the directions parallel and perpendicular to $\mathbf{B}$, respectively, and $\nabla_{\theta \varphi}$ is the gradient in velocity space at $v=$ const. The transport 
coefficients $D$ and $K$ describe pitch-angle scattering and field line random walk, respectively. The scattering rate may be estimated from Voyager 1 measurements of magnetic fluctuations $[13,5]$. Assuming for simplicity isotropic scattering, the rate is calculated as

$$
D=D_{0} \frac{B}{B_{0}} \frac{Q}{\gamma A},
$$

where $Q$ is the charge number, $A$ is the mass number, $\gamma$ is the Lorentz factor, and $D_{0}$ is the reference value of the scattering coefficient that depends on the turbulent power. From Voyager data we are able to estimate $D_{0} \sim 10^{-4} \mathrm{~s}^{-1}$ in the inner heliosheath, while its value in the LISM is at least 100 times smaller.

Another key ingredient of the model is the presence of a latitudinal gradient of the magnetic field strength, shown with an arrow in Figure 2. This gradient results in a radial drift of gyrating ions with speed $v_{d} \sim r_{L} v_{\perp} /(2 L)$, where $r_{L}$ is the Larmor radius and $L$ is the scale length of the magneticfield variation. With $L=10 \mathrm{AU}$, the effect of drifts differentiates charged particles based on their Larmor radius (i.e., rigidity). For example, at the same energy per nucleon singly anomalous oxygen ions, which are primarily single charged, have 16 times the rigidity of protons, so these ions are able to penetrate much deeper into the LISM before they become scattered and escape along the field lines. The simulation size was about $2 \mathrm{AU}$ wide in the radial direction and $60 \mathrm{AU}$ wide in the azimuthal direction. The latter value is also the length of the flux tubes discussed in Section 2.

Figure 3 shows model-derived intensities of $5 \mathrm{MeV} / \mathrm{n}$ anomalous hydrogen, helium and oxygen ions near the heliocliff, which is located at $r=0$ in this figure. The prominent dropouts correspond to the interiors of the flux tubes, where particle escape into the LISM. Past the heliocliff, the streaming ions (circles) are depleted first with the resulting distribution becoming flattened near $\mu=0$. In the model, the intensity of singly charged oxygen ions remains relatively high for much longer than hydrogen, which is consistent with the Voyager observations [2].

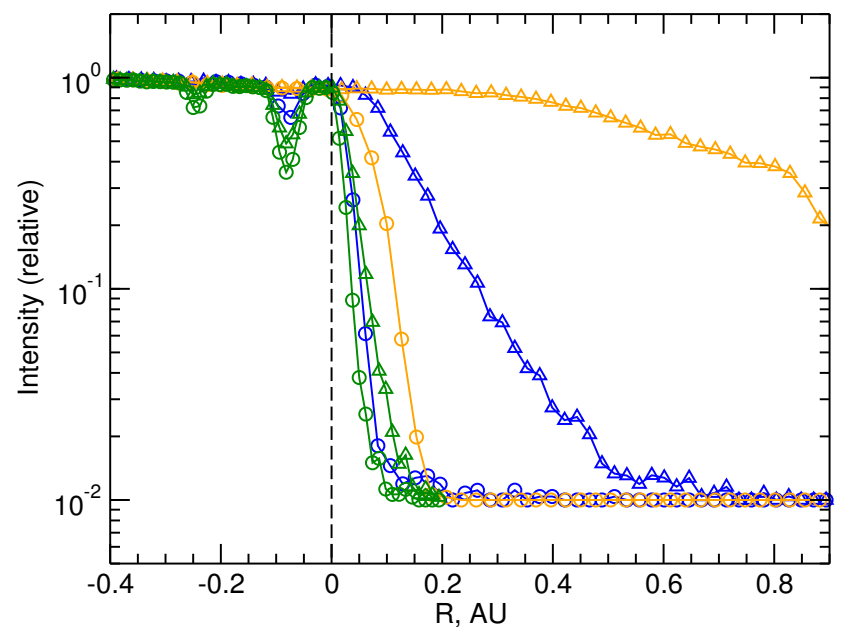

Figure 3: Intensities of anomalous protons (green), helium (blue) and oxygen (orange) as a function of radial distance from the heliocliff (the vertical dashed line). Circles show intensities of streaming ions, while triangles show intensities of ions gyrating near $90^{\circ}$.

The dropouts also offer a unique opportunity to estimate the speed of plasma flowing past Voyager 1 (whose plasma instrument is not functioning). Figure 4 compares the count rates of 2-8 $\mathrm{MeV}$ protons measured by two Voyager CRS sensors near the second dropout. The pointing of the CRS instrument sensors is such that one of them (LET B) is observing ions whose guiding centers 
lie closer to the sun, while another sensor (LET D) is measuring ions with guiding centers located farther away. When crossing a large intensity gradient, such as at a boundary of a flux tube, LET B temporal profile trails that of LET D by approximately the time it takes for the spacecraft to travel a distance of $2 r_{L}$ in the plasma frame. From Figure 4 one estimates the temporal delay to be about $13 \mathrm{hrs}$, which gives a range of radial plasma velocities of between $-5 \mathrm{~km} \mathrm{~s}^{-1}$ and $+1 \mathrm{~km}$ $\mathrm{s}^{-1}$, given the uncertainties in the direction of the flux tube axis. This very small radial speed is consistent with the indirect measurements of the velocity based on Compton-Getting anisotropy of low energy ions [14].

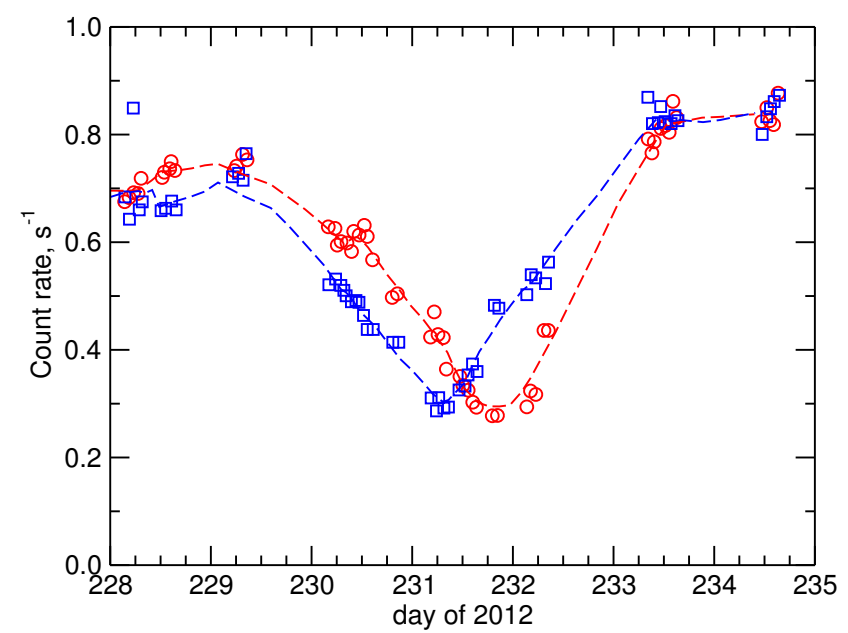

Figure 4: Count rates of $2-8 \mathrm{MeV}$ protons near the second intensity dropout. LET B (red) measures ions gyrating toward the Voyager from the sunward direction, while LET D (blue) measures ions gyrating in from farther away.

\section{Discussion}

Three years after the 2012 events at Voyager 1 it appears that the crossing of the heliopause was consistent with the theory predictions, for the most part (galactic and anomalous cosmic rays, magnetic field strength, turbulent fluctuations, plasma density). Certain magnetic field observations (in particular, an apparent absence of directional change at the heliocliff) generated a great deal of controversy, leading some $[15,16]$ to conclude that Voyager 1 crossed not the heliopause, but a different magnetic boundary still in the inner heliosheath. In fact, the direction did change by about $20^{\circ}$ gradually over 30 days prior to the heliocliff [6]. Global MHD models predict that the draping of the interstellar magnetic field lines around the heliopause could rotate the LISM field to within $10^{\circ}$ of the measured direction at the Voyager 1 location [17], see also [18, 19]. The proximity of the draped field direction to the azimuthal is therefore coincidental and limited to the direction Voyager 1 is traveling.

The complex evolution of the magnetic field prior to the heliocliff suggests that the heliopause is not a thin surface, but a region of some $0.3 \mathrm{AU}$ in width with the heliocliff as its leading edge, as measured from the LISM. We propose that this regions is permeated by turbulence-free LISM magnetic flux tubes with diameters of the order of $0.1 \mathrm{AU}$ and perhaps 50-100 AU in length that allow anomalous cosmic rays to escape the region by free streaming. For galactic cosmic rays, the same tubes would provide a more direct access to the inner heliosheath. It is essential that the magnetic fields inside and outside the tubes to be parallel, otherwise the interchange would 
not occur. The development of the instability may actually lead to such an alignment at selected locations if the initial magnetic shear is not too large.

The model presented here has the advantage of being consistent, on the quantitative level, with the observations and theoretical interpretations of the energetic particle intensities and pitch angle distributions before and after the heliocliff $[9,10]$. In the interchange scenario the flux tubes are connected to the LISM at both ends, and the energetic particles can escape in both direction with equal probability, so the remaining gyrating population develop a large second-order and only a modest first-order anisotropy, as observed [1,2]. The consistent time shift between the ACR intensities measured by two Voyager 1 LET telescopes supports the interpretation of the dropouts as flux tube crossings, rather than repeated encounters of a single moving boundary.

\section{Acknowledgments}

This work was supported, in part, by NASA grant NNX12AH44G and by NSF grant AGS-0955700.

\section{References}

[1] Krimigis, S. M., Decker, R. B., Roelof, E. C., et al. 2013, Science, 341, 144

[2] Stone, E. C., Cummings, A. C., McDonald, F. B., et al. 2013, Science, 341, 150

[3] Burlaga, L. F., Ness, N. F., and Stone, E. C. 2013, Science, 341, 147

[4] Gurnett, D.A., Kurth, W. S., Burlaga, L. F., and Ness, N. F. 2013, Science, 341, 1489

[5] Burlaga, L. F., Florinski, V., and Ness, N. F. 2015, Astrophys. J. Lett., 804, L31

[6] Burlaga, L. F., and Ness, N. F. 2014, Astrophys. J., 784, 146

[7] Swisdak, M., Drake, J. F., and Opher, M. 2013, Astrophys. J. Lett., 774, L8

[8] Strumik, M., Grzedzielski, S., Czechowski, A., Macek, W. M., and Ratkiewicz, R. 2014, Astrophys. J., 782, L7

[9] Florinski, V., Jokipii, J. R., Alouani-Bibi, F., and le Roux, J. A. 2013, Astrophys. J. Lett., 776, L37

[10] Florinski, V., Stone, E. C., Cummings, A. C., and le Roux, J. A. 2015, Astrophys. J., 803, 47

[11] Bateman, G. 1978, MHD Instabilities, MIT Press: Cambridge, MA

[12] Florinski, V. 2011, Adv. Space Res., 48, 308

[13] Burlaga, L. F., Ness, N. F., Florinski, V., and Heerikhuisen, J. 2014, Astrophys. J., 792, 134

[14] Krimigis, S. M., Roelof, E. C., Decker, R. B., and Hill, M. E. 2011, Nature, 474, 359

[15] Fisk, L. A., and Gloeckler, G. 2013, Astrophys. J., 776, 79

[16] Schwadron, N. A., and McComas, D. J. 2013, Astrophys. J. Lett., 778, L33

[17] Grygorczuk, J., Czechowski, A., and Grzedzielski, S. 2014, Astrophys. J. Lett., 789, L43

[18] Opher, M., and Drake, J. F. 2013, Astrophys. J. Lett., 778, L26

[19] Borovikov, S. N.,and Pogorelov, N. V. 2014, Astrophys. J. Lett., L16 